\title{
CFD Analysis of Mixing Characteristics of Several Fuel Injectors at Hypervelocity Flow Conditions.
}

\author{
Tomasz G. Drozda, ${ }^{*}$ J. Philip Drummond, ${ }^{\dagger}$ and Robert A. Baurle ${ }^{\ddagger}$ \\ NASA Langley Research Center, Hampton, VA, 23681
}

\begin{abstract}
CFD analysis is presented of the mixing characteristics and performance of three fuel injectors at hypervelocity flow conditions. The calculations were carried out using the VULCAN-CFD solver and Reynolds-Averaged Simulations (RAS). The high Mach number flow conditions match those proposed for the planned experiments conducted as a part of the Enhanced Injection and Mixing Project (EIMP) at the NASA Langley Research Center. The EIMP aims to investigate scramjet fuel injection and mixing physics, improve the understanding of underlying physical processes, and develop enhancement strategies and functional relationships relevant to flight Mach numbers greater than eight. Because of the high Mach number flow considered, the injectors consist of a fuel placement device, a strut; and a fluidic vortical mixer, a ramp. These devices accomplish the necessary task of distributing and mixing fuel into the supersonic cross-flow albeit via different strategies. Both of these devices were previously studied at lower flight Mach numbers where they exhibited promising performance in terms of mixing efficiency and total pressure recovery. For comparison, a flush-wall injector is also included. This type of injector generally represents the simplest method of introducing fuel into a scramjet combustor, however, at high flight Mach number conditions, the dynamic pressure needed to induce sufficient fuel penetration may be difficult to achieve along with other requirements such as achieving desired levels of fuel-to-air mixing at the required equivalence ratio. The three injectors represent the baseline configurations planned for the experiments. The current work discusses the mixing flow field behavior and differences among the three fuel injectors, mixing performance as described by the mixing efficiency and the total pressure recovery, and performance considerations based on the thrust potential.
\end{abstract}

\section{Introduction}

$\mathrm{R}^{\mathrm{R}}$ ECENT flight demonstrations of hypersonic air-breathing vehicles ${ }^{1,2}$ prove their increasing promise for military (rapid response and strike capability on a global scale), aerospace (safer and more affordable access to space), and civil aviation (hypersonic point-to-point transport) applications. However, designing engines capable of robust hypersonic air-breathing operation, characterized by rapid fuel-air mixing and short combustion times while ensuring flame stability over a wide range of speeds, has proven difficult. Attempts at improving fuel injection to enhance fuelair mixing while simultaneously reducing total pressure losses have received a great deal of attention over the years. Although some total pressure loss is thermodynamically unavoidable and occurs due to the desired effect of molecular mixing of the fuel and air, any losses beyond this minimum required amount reduce the thrust potential of the engine.

Some of the mixing enhancements considered in the literature include variable angle injection, non-circular injectors, and small and large vortex/swirl generating tabs and ramps. For high Mach number internal flows, where combustor residence times are increasingly small, and compressibility effects may further suppress mixing, ${ }^{3-8}$ poor injector performance leads to unnecessarily long combustor lengths, and therefore, increased drag and reduced thermal performance of the engine. In an effort to achieve more rapid mixing at high speeds, designers have typically considered multiport fuel placement devices, such as slender, often swept, struts protruding into the core flow, and/or large vortex generators, such as swept or unswept ramps that induce large axially-rotating vortices. A good overview

* Research Aerospace Engineer, Hypersonic Airbreathing Propulsion Branch, AIAA Senior Member.

${ }^{\dagger}$ NASA Distinguished Research Associate, AIAA Fellow

${ }^{\ddagger}$ Research Aerospace Engineer, Hypersonic Airbreathing Propulsion Branch, AIAA Associate Fellow. 
was recently provided by Lee et al. ${ }^{9}$ Ramp injectors rely on large vortices to entrain the air and fuel into one another, and enlarge and stretch the fuel-air interface area. In contrast, strut injectors typically generate smaller and weaker vortices and rely on fuel port features (such as fuel port shape and/or tabs) to enhance small-scale mixing within the developing fuel-air mixing layer. Both injector types also produce wakes, which contain slower moving air. Several ramps or struts are typically used to "fill" the combustor cross section in a manner that provides enough fuel to satisfy the equivalence ratio requirements. An additional benefit of strut and ramp injection, increasingly important in higher Mach number flows, is that the fuel can be easily injected parallel to the main air flow. In such a configuration, the momentum of the aft-facing fuel stream augments the thrust generated by the combustion processes. It should be noted that despite the small amount of fuel mass entering the engine, the thrust augmentation from aft-facing fuel injector ports may be significant, especially when the fuel is used for cooling of the various vehicle components. In those situations, the aft-facing fuel ports become energy recovery devices by converting heat absorbed in cooling the vehicle components into thrust. The strut and ramp injectors address various limitations of a flush-wall injector, which though typically induces less total pressure loss, suffers from fuel penetration and reduced mixing issues, which are further exaggerated when the injector is inclined to the flow to gain thrust augmentation (as discussed above).

This work focuses on comparative assessment of the mixing effectiveness of the three classes of injectors, namely a strut, a ramp, and an inclined flush-wall injector in a Mach 6 flow. Although the current work is CFD-based and could utilize true flight flow conditions, the configurations and conditions correspond to baselines proposed for the Enhanced Injection and Mixing Project (EIMP) at the NASA Langley Research Center. The EIMP aims to investigate scramjet fuel injection and mixing physics, improve the understanding of underlying physical processes, and develop enhancement strategies and functional relationships relevant to flight Mach numbers greater than eight. The experiments will utilize a "direct-connect," subcomponent-level approach with a Mach 6 facility nozzle to produce a flow field that simulates the non-distorted combustor entrance flow of a scramjet engine. However, although the value of the internal flow Mach number matches that expected at the entrance of the combustor in flight, the total enthalpies for these experiments are bounded between the condensation limit for the expanded facility air and the thermal-structural limits of the uncooled experimental hardware. For the EIMP experiments these limits correspond to about Mach 3.4 and 4.25 flight enthalpies, respectively. Furthermore, the fuel simulant for the mixing experiments will be helium, not hydrogen. The use of "cold" flows and non-reacting fuel simulants for mixing experiments is not new ${ }^{10}$ and has been extensively utilized as a screening technique for scramjet fuel injectors, and previously shown to be a good surrogate for the true flight environment by Drozda et al. ${ }^{11}$ The primary goal of the current work is not to pinpoint the best fuel injector for hypervelocity flow applications but rather to discuss physical considerations, and illustrate and highlight the many competing factors related to fuel injection that may impact the eventual injector performance at any flow conditions.

\section{Injector Geometries and Simulated Flow Conditions}

Three types of injectors were investigated in the current study. These are a strut, ramp, and rectangular, highaspect ratio flushwall injector. The strut and a ramp have been previously studied by Baurle et al. ${ }^{12}$ under "cold" flow conditions at a combustor entrance Mach number of 4.5. However, unlike the simulations of Baurle et al., ${ }^{12}$ which configured the injectors in a closed duct and interdigitated fashion, the current simulations include a row of injectors on an open flat plate. This is because an open flat plate is also used for the experimental configuration of the Enhanced Injection and Mixing Project (EIMP) in the NASA Langley Arc-Heated Scramjet Test Facility (AHSTF). While no experimental data is yet available, the EIMP experiments entail testing various fuel injection devices mounted on an open flat plate located downstream of a Mach 6 facility nozzle, which simulates a non-distorted combustor entrance flow of a flight vehicle traveling at a Mach number of about 14 to 16 . The flat plate is 28.87 inches long tip-to-tail with the fuel injection plane located at 8.87 inches downstream from the leading edge of the plate. Further details about the experimental setup and the EIMP are presented in Cabell et al. ${ }^{13}$ The current experiments focus on studying fuel injection and mixing processes in the absence of heat release by utilizing helium as the fuel simulant. Furthermore, these experiments are referred to as "cold" because the total temperature of the facility air is bounded between the condensation limit for the expanded facility air and the thermal-structural limits of the uncooled test hardware. The former and latter correspond to 728 and $978 \mathrm{~K}$, respectively, and are both significantly lower than the total temperature expected under flight conditions.

The first injector is a slender swept strut protruding into the flow. Strut injectors have several advantages in hypervelocity flow applications. First, they can be designed to place the fuel where it is needed, thereby alleviating the need to consider fuel penetration issues and focusing only on the injector spacing. Second, the injector ports on a strut 


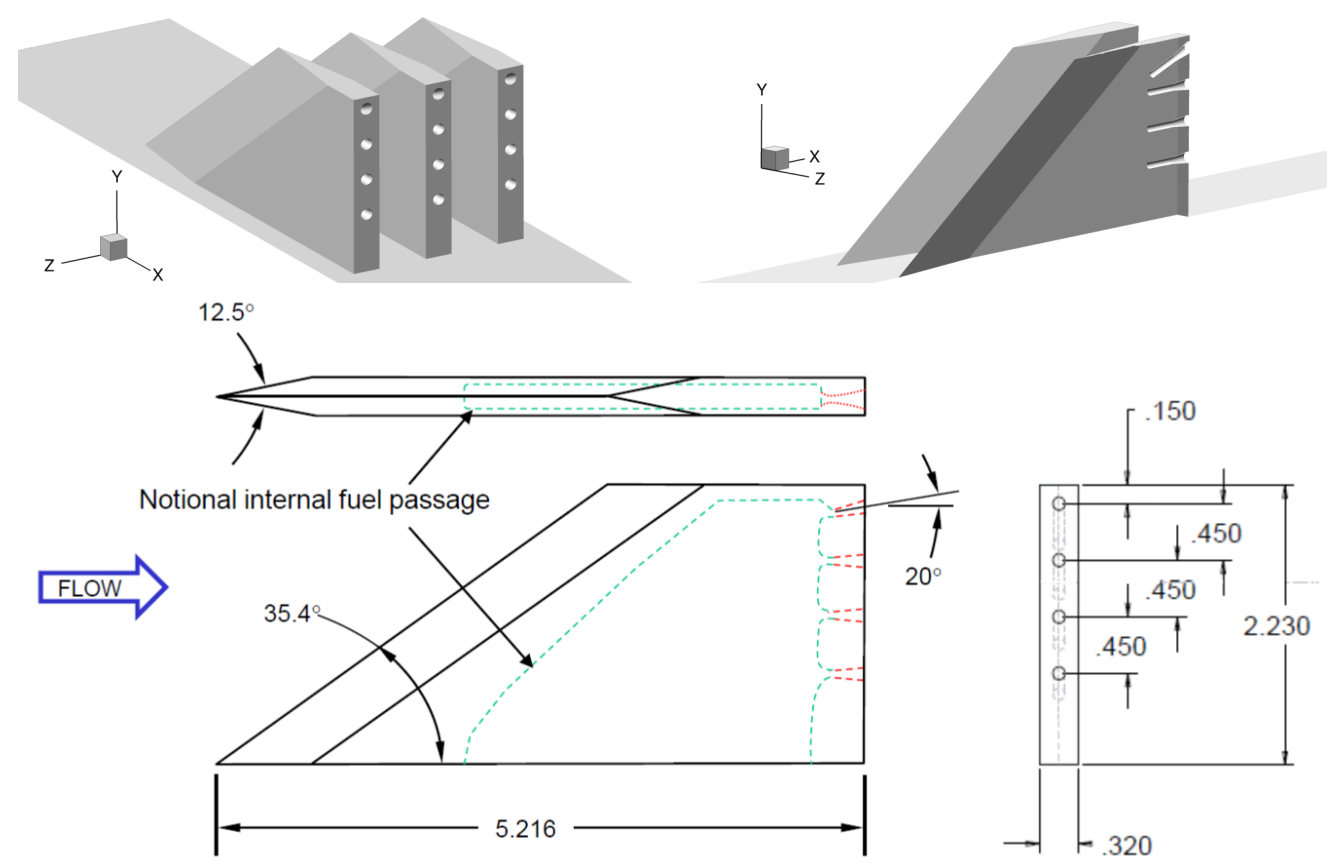

Figure 1. Isometric views and dimensional details of the baseline strut injector. Linear dimensions are in inches.

are typically aligned parallel to the flow, which allows for the injected fuel streams to augment the thrust of the engine. The potential downsides of a strut injector are the structural integrity and cooling requirements needed for its slender body, the drag (both viscous and pressure) that it induces on the flow by the obstruction it generates, and the total pressure loss incurred by the oblique shocks that emanate from its leading edge. The pressure drag could be reduced if the pressure at the trailing edge of the injector (i.e, injector base) is increased (say, by combustion). The viscous drag is proportional to the injector surface area exposed to the flow, which for a single strut injector is about 16.7 square inches. The area projected in the flow direction is 0.71 square inches. The shock waves introduce total pressure losses, pass through and interact with one-another, and reflect off the sidewalls of the combustor. The interactions of shock waves with the sidewall boundary layers has the potential to separate the flow and introduce local hot spots, as well as, additional flow blockage. However, these shock waves also introduce mixing enhancement via the effect of the baroclinic torque as they pass through the variable density interfaces of fuel-air mixing plumes and introduce localized vorticity. Views and dimensional details of the strut, are shown in Fig. 1. The $\mathrm{x}, \mathrm{y}$, and $\mathrm{z}$ denote the streamwise or downstream, vertical or wall normal, and cross-stream directions, respectively. In the current simulations, the struts are placed 0.9 inches apart. This spacing is the same as that found between the interdigitated strut injectors of Baurle et al. ${ }^{12}$ Each strut injector has four fuel injector ports. This is different from the interdigitated struts of Baurle et al., ${ }^{12}$ which only have three injector ports. The second injector port from the bottom is not needed in the interdigitated configuration because the staggered injector port of the adjacent strut is relied upon to fill this portion of the cross sectional area with fuel. Each injector port has a throat diameter of 0.083 inches followed by a conical expansion area with a half-angle of 6 degrees that is designed to expand hydrogen to an exit Mach number of about 2.6 and helium to Mach number of about 3.

The second injector is an unswept ramp. Compared to the strut, the ramp injector does not protrude as far into the flow but is wider and therefore, can introduce as much or more blockage. The surface, and flow-projected areas of the ramp injector are about 7.9 and 0.9 square inches, respectively. The former is proportional to the viscous losses, the latter to the extent of the downstream wake. The ramp injector generates a counter-rotating vortex pair (CVP) on each side of the ramp as the flow passes over the ramp and through the gaps between the ramps, which convects the injected fuel upwards, towards the core of the flow, and fills the intended fueling area. In addition to inducing the upward entrainment, the CVP stretches the fuel-air interface thereby increasing the surface area over which the molecular viscosity can act to mix the fuel and air. Because the strength of the CVP depends on both the geometry of the ramp, 


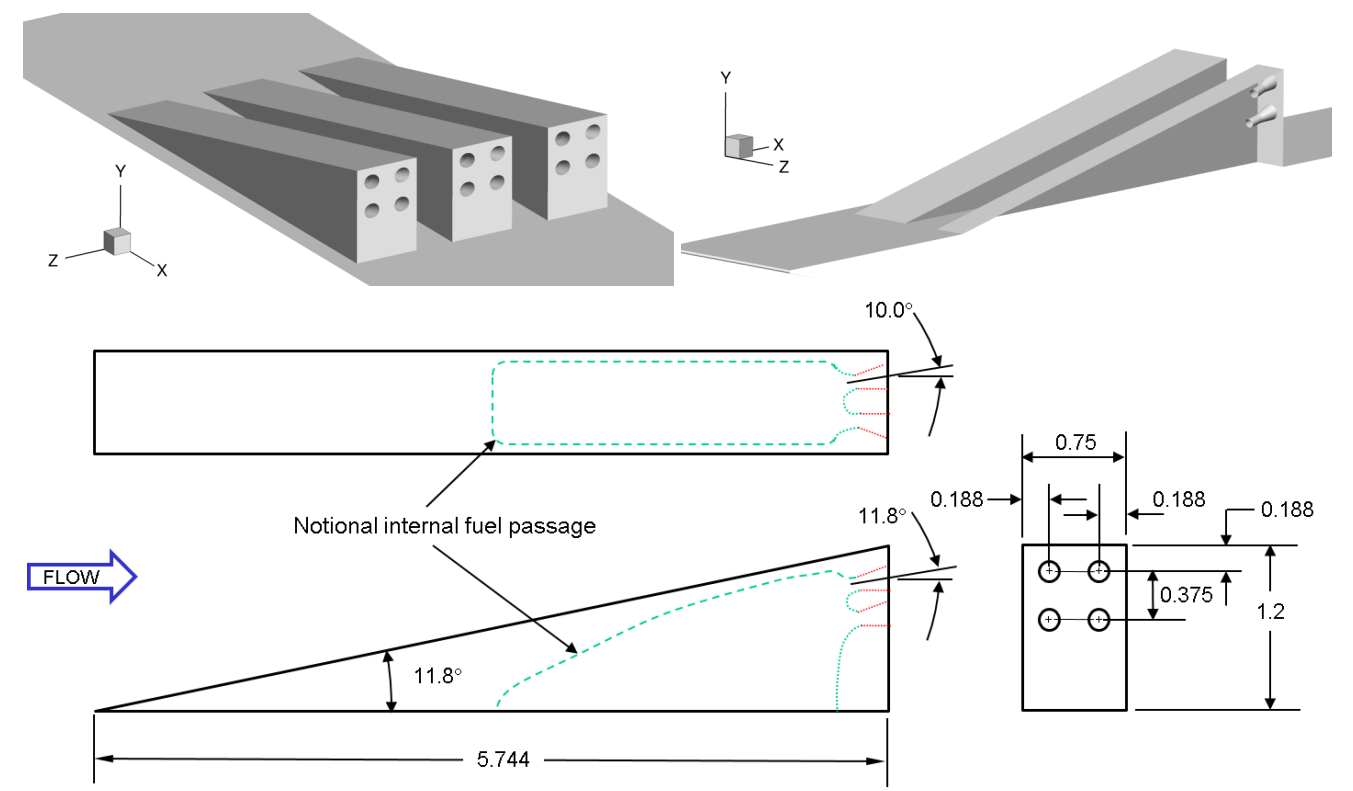

Figure 2. Isometric views and dimensional details of the baseline ramp injector. Dimensions are in inches.

and the incoming air flow conditions, designing an injector that robustly fills its intended fueling area across a range of flight conditions is more challenging for a ramp than a strut. As with the strut, the ports of the ramp injector are also nearly aligned parallel to the flow and allow for injected fuel streams to augment the thrust of the engine. However, the ports are angled slightly up and to the side to aid in directing the fuel streams toward the CVP. The ramp injector also generates an oblique shock due to its inclined surface. As is the case for the shock waves generated by the strut injector leading edge, the ramp shock also induces total pressure losses, and reflects off the top and/or bottom surfaces of the flowpath where it interacts with the boundary layer and can separate it inducing further losses, increased heat transfer to the wall, and flow blockage. Because the ramp induces only a single oblique shock, the total pressure losses are expected to be smaller than those of the struts, but the potential for mixing enhancement via the baroclinic torque is also reduced. Views of the ramp, and its dimensional details are shown in Fig. 2. In the current simulations, the adjacent ramps are placed 1.2 inches apart. This spacing is also the same as that found between the interdigitated ramp configuration of Baurle et al. ${ }^{12}$ Each injector port has a throat diameter of 0.108 inches followed by a conical expansion area with a half-angle of 10 degrees that is designed to expand hydrogen to an exit Mach number of about 2.6 and helium to a Mach number of about 3.

The third injector is a high-aspect-ratio rectangular flushwall injector. Unlike the strut and ramp, the flushwall injector introduces no physical blockage into the flow. Instead, a number of flow features form around the injection site that interact to produce a similar effect. These features are shown schematically for a generic round flushwall injector in Fig. 3. The bow shock that forms upstream of the injection plume creates both total pressure losses and aerodynamic blockage by forcing the air stream to flow around the fuel plume. As is the case for the ramp injector, the fuel plume entering the high-speed cross flow generates a CVP, which becomes the main mechanism for stirring the fuel into the air. However, unlike a fuel placement device, such as a strut, the extent to which the fuel penetrates into the air flow is governed by fluidic considerations. ${ }^{9,15-17}$ The jet penetration has been shown to be primarily proportional to the dynamic pressure (or momentum flux) ratio between main air and the fuel jet, and is further enhanced by matching the static pressure at the exit of the fuel injector to the effective static pressure, that is, the static pressure of the air just upstream of the fuel plume and downstream of the bow shock. Penetration also increases with the increasing thickness of the approach boundary layer ${ }^{18}$ due to aerodynamic "shielding." These observations led to the development of the aero-ramp ${ }^{19}$ and the cascade injectors, ${ }^{20}$ which utilized an array of smaller jets, carefully designed to take advantage of both shielding and pressure matching to enhance the fuel jet penetration. Decomposing the area of a single large flushwall injector into multiple smaller ones has the additional advantage of physically increasing the fuel-air interface area, which enhances mixing. For example, the fuel-air interface area increases by a factor of $\sqrt{N}$ when a large area 

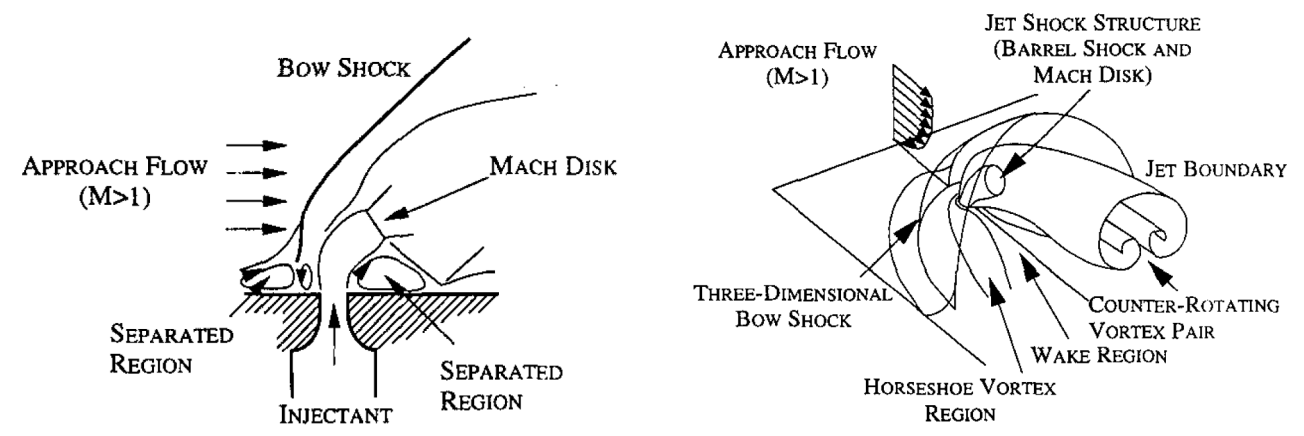

Figure 3. Side and isometric views of the flow features that form around a generic flushwall injector during transverse injection of fuel into the supersonic cross-stream. From Gruber et al. ${ }^{14}$

is decomposed into $N$ equal parts. Nevertheless, even these advanced concepts have limited application when scaling to larger flowpaths and operating at off-design conditions.

The flushwall injector exit geometry is based on the multi-objective optimization work of Ogawa ${ }^{21}$ whose approach using a genetic algorithm revealed four families of high performing flushwall injectors. The injector port chosen for the current work has a constant width, rectangular cross-section, with an aspect ratio of 8 at the injector exit plane, and the longer dimension aligned in the streamwise direction. The injector is also inclined at 30 degrees to the wall. The isometric views of the flushwall injector are shown in Fig. 4. The injector has been further designed to qualitatively match the geometrical features of the fuel ports of the strut injector. As such, the flushwall injector contains an expansion section with a 6 degrees half-angle, and an expansion area ratio matching that of the strut conical fuel port. The area at the end of the expansion section, but before the 30 degrees rotation, has been adjusted to match the total exit area of the 4 fuel ports of the strut. The width of the injector is 0.1392 inches with a throat height of 0.1541 inches. The adjacent flushwall injectors are placed 1.704 inches apart. This spacing corresponds to about 6 times the diameter of a circular injector with an equivalent area, and about 1.5 times the length of the current injector, allowing for a sufficient air-gap between the adjacent injectors even after the expected axis-switching of the fuel plume. ${ }^{21}$

It should be noted that because the current simulations are for an open plate configuration, they do not take into account the area expansion that is sometimes used to relieve the area blockage introduced into a ducted flowpath by struts, ramps, or the aero-dynamic effects of the flushwall injection. In addition to counteracting the area blockage, this area relief causes the flow to expand through the gaps between the injectors and further enhance the strength of the CVP for the ramp configurations. Furthermore, the area expansion naturally ends at the base of the injector where the combustor walls typically turn parallel again (or continue at a very shallow angle of up to few degrees). This turning of the flow induces a shock wave at the base of the injector, which introduces additional total pressure losses but also enhances the fuel-air mixing via baroclinic torque effects, as discussed earlier. However, although potentially beneficial with respect to the mixing enhancement, the use of any flow expansions inside the combustor operating at high Mach numbers would lead to increased Rayleigh losses due to chemical reactions because the heat addition would occur at a higher value of the Mach number. Furthermore, the lower combustor static pressure could impact the combustion efficiency in a way that might offset any benefits associated with mixing enhancement.

The inflow conditions correspond to a total pressure and total temperature of $4.31 \mathrm{MPa}(625 \mathrm{psi})$ and $978 \mathrm{~K}$ $\left(1760{ }^{\circ} \mathrm{R}\right)$, respectively, expanded to a Mach number of 6.36. A non-reacting, thermally perfect mixture of $21 \%$ oxygen $\left(\mathrm{O}_{2}\right), 78 \%$ nitrogen $\left(\mathrm{N}_{2}\right)$, and $1 \%$ nitric oxide $(\mathrm{NO})$ by volume was used for the air. A small amount of NO was present to account for production of this species in the experimental facility, ${ }^{22}$ although its impact on the current simulations is expected to be negligible. The fuel mass flow rate of helium for each injector was computed by assuming an equivalence ratio of one over the intended fueling area as if it were fueled with hydrogen. An intended fueling area is established independently for each injector. For example, a combustor cross-section area fueled with slender struts will likely require such devices to be spaced more closely than a similar combustor fueled with large vortex generating ramps. Therefore, the intended fueling area will be narrower for struts than for the ramps. The intended fueling areas for the strut and ramp are obtained from Baurle et al. ${ }^{12}$ who considered them in a realistic scramjet combustor configuration. Since the flushwall injector was designed with the intent to fuel the same flowpath, the intended fueling area height for the flushwall injector is the same as that for the strut with the width obtained from the optimization 


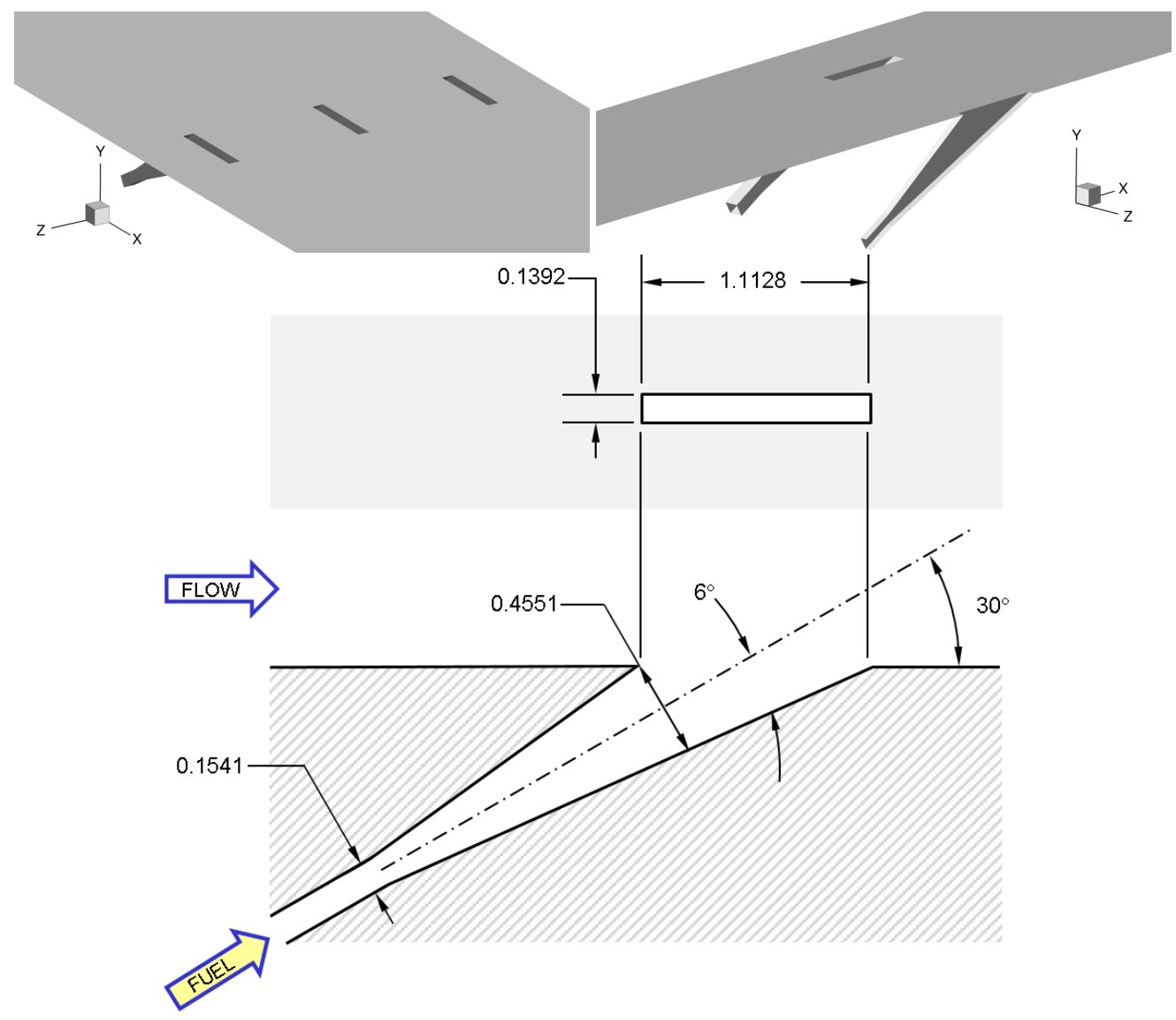

Figure 4. Isometric views and dimensional details of the baseline flushwall injector.

work of Ogawa. ${ }^{21}$ The details of all of the flow parameters used in the current simulations are shown in Table 1. Both the properties of air and fuel are presented. The subscripts $f$ and $a$ denote fuel and air flow streams, respectively. In addition to the quantities needed for the simulations, a few nondimensional quantities are also shown; these are, the unit Reynolds numbers per inch for the air and fuel streams, velocity difference parameter, $\Delta \mathrm{U}$, the convective Mach number, $\mathrm{M}_{\mathrm{c}}$, and the ratios of the density, $\rho_{\mathrm{f}} / \rho_{\mathrm{a}}$, static pressure, $\mathrm{p}_{\mathrm{f}} / \mathrm{p}_{\mathrm{a}}$, and dynamic pressure, $J$ between the fuel and air stream. All values are computed based on the combustor entrance flow conditions for the air and the expanded flow conditions at the exit of the injector ports for the fuel. The nondimensional quantities have been found to be relevant to the injection and mixing processes in canonical problems. ${ }^{3,23,24}$

\section{Metrics of Interest}

A number of different metrics for thrust performance, mixing and combustion efficiency, and associated thermodynamic losses exist with the most detailed analysis proposed by Riggins et al. ${ }^{25}$ For the current study, the following were chosen: the integrated (in the cross-stream and vertical directions) forms of the total pressure recovery, mixing efficiency based on stoichiometric proportions of fuel and air, and thrust potential. The total mass-flux-weighted pressure recovery is defined as:

$$
P_{t}^{r e c}=\frac{\int P_{t} \rho U d A}{\int P_{t_{\infty}} \rho U d A}
$$

where $P_{t}$ is the total pressure, $\rho$ is the density, $U$ and $d A$ are the velocity magnitude and the incremental area projected in the streamwise direction, respectively. This parameter is proportional to the difference between sensible entropies computed at the total and static values of the temperature, and therefore, gives a measure of the thermodynamic 
Table 1. Global parameters of interest for the strut, ramp, and flushwall injector configurations. The last set of rows contain ratios of interest between the fuel (helium) and air streams, where the subscripts $f$ and $a$ denote fuel and air streams, respectively.

\begin{tabular}{|c|cccc|}
\hline Property & Air $^{\dagger}$ & Strut & Ramp & Flushwall \\
\hline Mach & 6.356 & 2.977 & 2.956 & 2.977 \\
$P 0(\mathrm{MPa})$ & 4.309 & 0.224 & 0.0882 & 0.424 \\
$T 0(\mathrm{~K})$ & 977.8 & 293.15 & 293.15 & 293.15 \\
$P(\mathrm{kPa})$ & 1.808 & 7.205 & 2.9114 & 13.642 \\
$T(\mathrm{~K})$ & 112.4 & 74.14 & 74.91 & 74.14 \\
$\mathrm{u}(\mathrm{m} / \mathrm{s})$ & 1352.6 & 1508.2 & 1505.6 & 1508.2 \\
$R e /$ in $\times 10 \mathrm{e} 6$ & 315.0 & 352.6 & 139.8 & 667.6 \\
\hline $\mathrm{Area}$ & & 0.9 in $\times 2.25$ in & 1.2 in $\times 2.25$ in $/ 2$ & 1.704 in $\times 2.25$ in \\
$\mathrm{m}_{\mathrm{a}}(\mathrm{kg} / \mathrm{s}) \times 10 \mathrm{e}-3$ & & 98.76 & 65.84 & 187.00 \\
$\mathrm{~m}_{\mathrm{f}}(\mathrm{kg} / \mathrm{s}) \times 10 \mathrm{e}-3$ & & 2.884 & 1.922 & 5.460 \\
\hline$\Delta \mathrm{U}^{\S}$ & & 0.0544 & 0.0535 & 0.0544 \\
$\mathrm{M}_{\mathrm{c}}$ & & 0.2163 & 0.2119 & 0.2163 \\
$\rho_{\mathrm{f}} / \rho_{\mathrm{a}}$ & & 0.8371 & 0.3348 & 1.5849 \\
$\mathrm{p}_{\mathrm{f}} / \mathrm{p}_{\mathrm{a}}$ & & 3.9845 & 1.6100 & 7.5440 \\
$J^{\|}$ & & 1.0408 & 0.4149 & 1.9706 \\
\hline
\end{tabular}

\footnotetext{
${ }^{\dagger} 21 \% \mathrm{O}_{2}, 78 \% \mathrm{~N}_{2}, 1 \% \mathrm{NO}$

${ }^{\ddagger}$ Intended fueling area for the injector

$\S$ Velocity difference parameter, $\Delta U=\left(u_{f}-u_{a}\right) /\left(u_{f}+u_{a}\right)$

${ }^{\top}$ Convective Mach number, $M_{c}=\left|u_{f}-u_{a}\right| /\left(c_{f}+c_{a}\right), c$ denotes the speed of sound.

"Dynamic pressure ratio, $J=\left(\rho_{f} u_{f}^{2}\right) /\left(\rho_{a} u_{a}^{2}\right)=\left(\dot{m}_{f} u_{f}\right) /\left(\dot{m}_{a} u_{a}\right)$.
}

losses. For purely mixing simulations, the total pressure recovery quantifies the losses due to the drag on the injector bodies and the surface of the flat plate, the mechanical stirring induced by injector bodies (especially the ramp), the turbulence, the molecular mixing, and shock wave losses. For reacting simulations, the total pressure recovery is further reduced by the chemical reactions, therefore, the values of the total pressure recovery obtained from the nonreacting simulations could be thought of as the maximum achievable for a given injector. Because chemical reactions reduce the total pressure recovery, which can be interpreted as a loss, yet energize the flow via heat release, which can be expanded into thrust, there is a need for another metric that more objectively quantifies the potential performance of the flowpath. The most direct metric that can achieve this is the thrust potential. This metric is obtained at each streamwise location by first one-dimensionalizing the flow at that location, and then allowing it to expand isentropically through an ideal thrust nozzle. In the current work, this thermodynamic process is evaluated until the flow reaches its combustor entrance value of the static pressure. The thrust potential is defined here by

$$
T P(N)=\dot{m} u_{e x i t}+\left(p_{\text {exit }}-p_{3}\right) A_{\text {exit }}
$$

where TP is the thrust potential, and $\dot{m}, u_{\text {exit }}, p_{\text {exit }}, A_{\text {exit }}$, and $p_{3}$ are the mass flow rate, velocity, pressure, and the area at the thrust nozzle exit plane, and the combustor entrance static pressure, respectively. Since the flow is expanded to the combustor entrance static pressure, the second term in the above equation is identically zero. Therefore, this metric represents the momentum portion of the ideal potential thrust that could be obtained when a flowpath of interest is truncated at a given streamwise location and coupled at that location to an ideal thrust nozzle. All of the losses in the value of the total pressure previously discussed for the purely mixing case would still appear as a decrement to the value of this thrust potential, however, the combustion heat release will increase the value of the thrust potential. It should be noted that the definition of the thrust potential is different from one used commonly when assessing vehicle performance. This is because we are simulating only a portion of the vehicle flowpath for which it is difficult to define a meaningful thrust, yet we are interested in a metric that would be proportional to it.

The mixing efficiency is defined in this work following Mao et al.: ${ }^{16}$

$$
\eta_{m}=\frac{\int \alpha_{R} \rho U d A}{\int \alpha \rho U d A}
$$


where the integration is over a single streamwise, $\mathrm{x}$-plane of interest, and $\alpha$ is the fuel or oxidizer mass fraction depending on whether the global equivalence ratio is less than or greater than 1, respectively. Quantity $\alpha_{R}$ is defined as the amount of fuel or oxidizer that would react if complete reaction took place without further mixing, i.e.,

$$
\alpha_{R}= \begin{cases}\alpha, & \alpha \leq \alpha_{s t} \\ \frac{\alpha_{s t}}{1-\alpha_{s t}}(1-\alpha), & \alpha>\alpha_{s t}\end{cases}
$$

where $\alpha_{s t}$ is the stoichiometric value of fuel or oxidizer mass fraction. For cases with an overall equivalence ratio of one, either fuel or oxidizer can be used in place of $\alpha$. But choosing fuel has a minor benefit of clarifying somewhat the meaning of Eq. (4), which becomes:

$$
\alpha_{R}= \begin{cases}Y_{f}, & Y_{f} \leq Y_{f, s t} \\ F A R_{s t} Y_{a}, & Y_{f}>Y_{f, s t}\end{cases}
$$

where $Y$ denotes mass fraction, $F A R_{s t}$ is the stoichiometric fuel to air ratio set to 0.0292 in the current work, and subscripts $f$ and $a$ denote fuel and air streams, respectively. It is clear from the above equation that if the local value of the mass fraction of fuel is less than its stoichiometric value, then that amount is "counted" as fully mixed because there is sufficient amount of air to completely deplete the fuel if reactions were allowed. However, if the local value of the fuel mass fraction is greater than its stoichiometric value, then the only part that could react is that which is in stoichiometric proportion to the local value of the mass fraction of the air. Therefore, only that portion is counted as being mixed in Eq. (3). The stoichiometric value of the hydrogen mass fraction is 0.0285 . The mixing efficiency formula of Mao et al. ${ }^{16}$ can also be used to analyze mixing in the reacting simulations, however, since fuel and oxidizer are consumed to make combustion products, care must be taken to consider the elemental mass fractions of fuel or oxidizer (i.e., mass fractions of all elements that originate in either fuel or oxidizer streams).

\section{Numerical Considerations}

The numerical simulations were performed using the Viscous Upwind aLgorithm for Complex flow ANalysis (VULCAN-CFD) code $^{26}$ VULCAN-CFD is a multi-block; structured-grid, cell-centered, finite-volume solver widely used for all-speed flow simulations. For this work, Reynolds-averaged simulations (RAS) were performed. The advective terms were computed using a Monotonic Upstream-Centered Scheme for Conservation Laws (MUSCL) scheme ${ }^{27}$ with the Low-Dissipation Flux-Split Scheme (LDFSS) of Edwards. ${ }^{28}$ The thermodynamic properties of the mixture components were computed using curve fits of McBride et al. ${ }^{29}$ The governing equations were integrated using an implicit diagonalized approximate factorization (DAF) method. ${ }^{30}$ The current work used the baseline blended $\mathrm{k}-\omega / \mathrm{k}-\epsilon$ turbulent physics model of Menter. ${ }^{31}$ The Reynolds heat and species mass fluxes were modeled using a gradient diffusion model with turbulent Prandtl and Schmidt numbers of 0.9 and 0.5 , respectively. Wilcox wall matching functions ${ }^{32}$ were also used, however, their implementation in VULCAN-CFD includes a modification that allows the simulations to recover the integrate-to-the-wall behavior as the value of $\mathrm{y}+$ approaches one. All simulations were converged until the total integrated mass flow rate and the total integrated heat flux on the walls remained constant. This typically occurred when the value of the $\mathrm{L}_{2}$-norm of the steady-state equation-set residual decreased by about 4-5 orders of magnitude. To conserve the available computational resources, all the simulations were split into an elliptic and a space-marching region. The elliptic region contained the inflow of the domain, the injector bodies, and up to 6.5 inches downstream of the injection plane. The computational cell count was about equal in both regions, but the computational cost associated with solving the space-marching regions was about an order of magnitude lower than that for the elliptic region. A single, fully elliptic simulation on a coarse grid confirmed that this approach indeed did not have a significant impact on any of the flow features nor the integrated values of the metrics of interest discussed in the previous section.

Three grids, coarse, medium, and fine, each progressively finer by a factor of 2 in each of the three dimensions, were used. The grid resolutions are summarized for the three injector types in Table 2. For the strut injector, a single full strut injector was included in the computational domain. This was done to help alleviate some of the grid skewness issues at the top of the strut, and near the leading edge of the strut. For the ramp injector case, only half of the ramp injector was included in the computational domain, taking advantage of the symmetry to reduce the grid size requirement. Similarly, the flushwall injector simulations also used only half of the full domain. All grids were generated with GridPro in the vicinity of the injector bodies and the leading edge of the flat plate, and 
further combined with Pointwise-generated h-blocks to complete the computational definition of the geometry. For all injectors, the inflow and outflow planes are placed 9 inches upstream, and 25 inches downstream of the fuel injection plane, which is located at $x=0$. Since both the inflow and the outflow consist of supersonic flow, static values of the temperature and pressure, and the Mach number are specified at the inflow; and all flow variables are extrapolated at the outflow. The vertical dimension is 6 inches high, which approximates the size of the EIMP facility nozzle core flow. ${ }^{33}$ Slip wall boundary conditions are used for the upper boundary of the flow domain. This was done to account for the presence of the facility nozzle jet stretchers used in the EIMP experiments. Since the boundary layer along the jet stretchers is of little interest and is not expected to impact the mixing region, no attempt was made to resolve and model it in the current simulations. Since the simulation domain effectively includes an infinite row of injectors in the cross-stream, the current simulations model the open plate experiment as a wide duct. In this model duct, the flow blockage due to injector bodies is $14 \%$ and $12.5 \%$ for the strut and ramp injectors, respectively. With the exception of the fuel ports, the grid was clustered towards all of the walls with the growth rates varying from $5 \%-15 \%$. The grid resolution study for the strut and ramp grids used in the current simulations have been previously reported by Drozda et al. ${ }^{11}$ The values of $y+$ for these cases, obtained on a fine mesh, vary up to about 20 , with the largest values observed on the injector bodies and fuel port walls. The y+ values along the flat plate are all less than one. The grid for the flushwall injector was built using the same density and near-wall spacing requirements as those for the strut and ramp, therefore the $y+$ values match those observed for the other cases. The values of $y+$ are about two and four times larger for the medium and coarse meshes, respectively.

The one-dimensional values of the total pressure recovery and the mixing efficiency versus the downstream distance in inches obtained from the simulations on the coarse, medium, and fine meshes are shown in Fig. 5. Due to the limitation of the one-dimensional post-processor to analyze only axial planes of data, some plots contain gaps where the complicated grid topology contained streamwise grid "wraps". These "wraps" could have been interpolated onto a Cartesian mesh, however numerical interpolation of cell center quantities could have introduced additional errors that would be external to the solver. To avoid this, the "wrap" regions were omitted from the one-dimensional analysis. In addition, the grid independence study for the strut and ramp injectors were previously performed ${ }^{11}$ under different flow conditions. Because these flow conditions corresponded to the higher values of the Reynolds numbers for both the main air flow and the fuel ports, the study was deemed conservative with respect to the current conditions. But, because of the flow differences, the profiles for the mixing efficiency and the total pressure recovery for the strut and ramp injectors reported in this section are shifted from those reported in the results section. The grid independence study shown for the rectangular injector has been performed under the flow conditions reported in Table 1. In addition, unlike the strut and ramp injectors, which introduce all of the fuel at a single downstream location, the flushwall injector has a downstream length over which the fuel is effectively distributed. This feature manifests itself in the one-dimensional plot as a rapid rise and decline in the value of the mixing efficiency because the value of the total integrated mass flow rate of the fuel gradually increases from the beginning of the injection plane until the downstream edge of the flushwall injector.

The one-dimensional values of the total pressure recovery and the mixing efficiency change monotonically with increasing grid resolution. However, the rate of convergence at which these values approach their asymptotic state varies among the cases and with the downstream distance. This behavior is possibly due to the use of wall matching functions on the injector bodies and inside the fuel ports. The behavior of these functions and the resulting boundary layers change for different grid densities as the $y+$ values change. The error bars corresponding to the data obtained on the fine mesh and shown in Fig. 5 were obtained assuming a unity order of accuracy and using the Grid Convergence Index (GCI), ${ }^{34}$ which is based on Richardson extrapolation. The resulting error bars are proportional to the difference between the results obtained on the fine and medium meshes, and represent an estimate of the error bounds between the current result and its fully grid-converged value. Although the formal order of accuracy of VULCAN-CFD is second order, unity order of accuracy was used for the GCI to ensure a conservative estimate of the errors. Furthermore, visual inspection of the differences between the one-dimensional values of the mixing efficiency, obtained from the

Table 2. Grid densities used in the current simulations.

\begin{tabular}{c|c|c|c|} 
& Strut & Ramp & Flushwall \\
Coarse & $4,921,682$ & $4,057,382$ & $4,356,936$ \\
Medium & $39,373,456$ & $32,459,056$ & $34,855,488$ \\
Fine & $314,987,648$ & $259,672,448$ & $278,843,904$
\end{tabular}



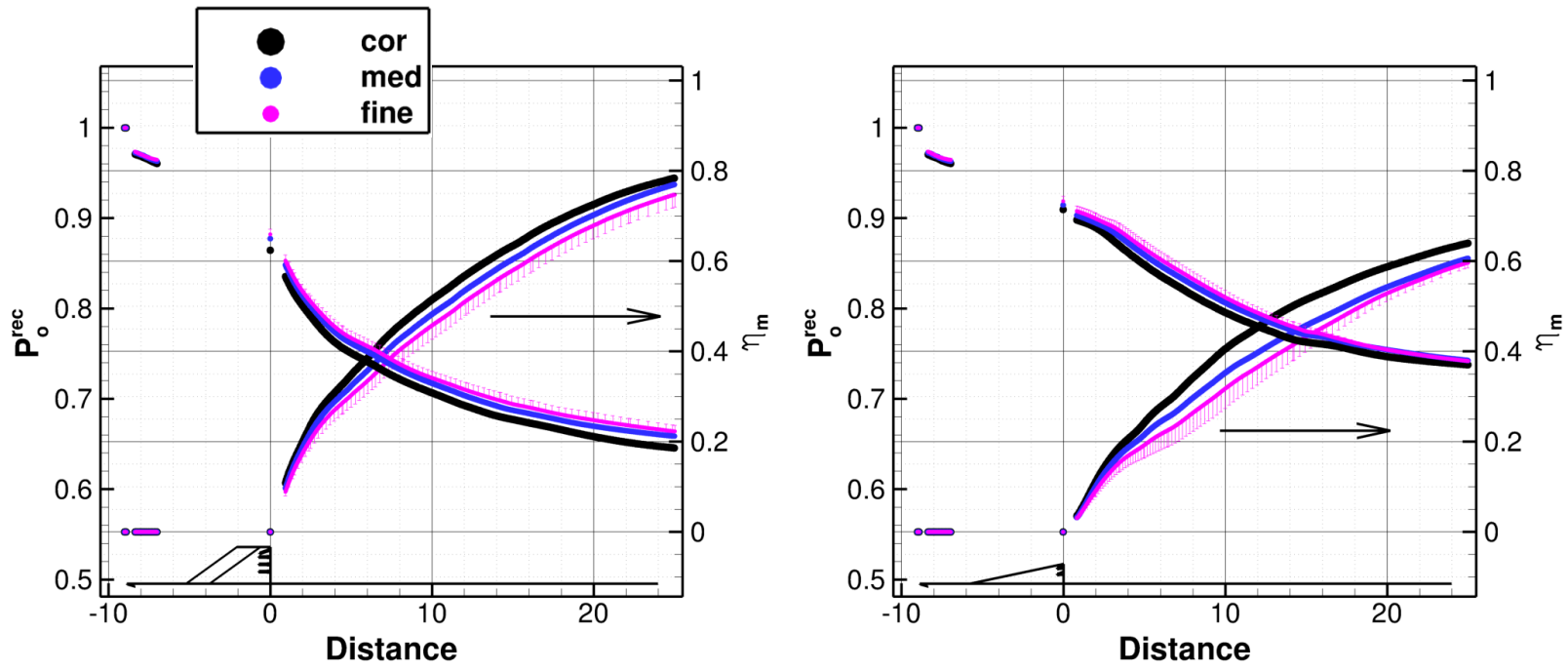

(c) Flushwall

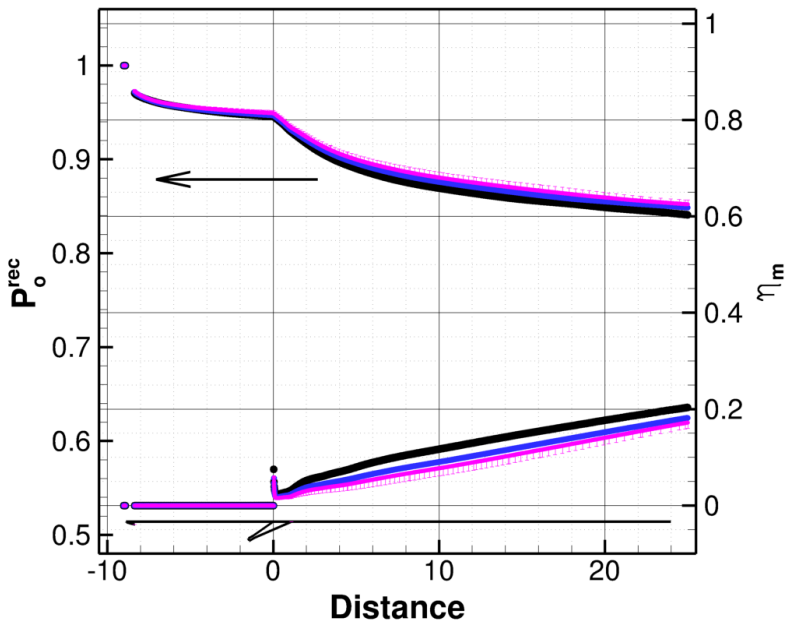

Figure 5. One-dimensional values of the total pressure recovery and mixing efficiency vs. downstream distance (in inches) obtained from the simulations on the coarse, medium, and fine grids, for the cases corresponding to the strut, ramp, and flushwall injectors.

simulations on the three different grids, reveals that these data appear to be converging slower than the formal order of accuracy of the solver. It should be noted that this result is not a reflection of the formal order of accuracy of the VULCAN-CFD solver, but rather the impact of shocks on the flow field. To ensure smooth solution near the flow discontinuities and improve the simulation stability, the solver reverts to the unity order of accuracy near these discontinuities. The observed numerical errors in the values of the total pressure recovery have a maximum value of about 5\%, with 1\%-2\% typical, and a maximum value of about $15 \%$ (in the near-field of the ramp injector case) with $5 \%$ typical for the mixing efficiency. Because the errors in the $1 \mathrm{D}$ values of the quantities of interest approach limits typically encountered in the engineering analysis, only the results of the simulations obtained on the fine meshes are presented in the subsequent sections.

All of the current simulations were performed on the Pleiades Supercomputer located at NASA Ames. The computational cost associated with solving a single simulation on the fine mesh was about $60 \mathrm{k}$ CPU hours. Simulations, 

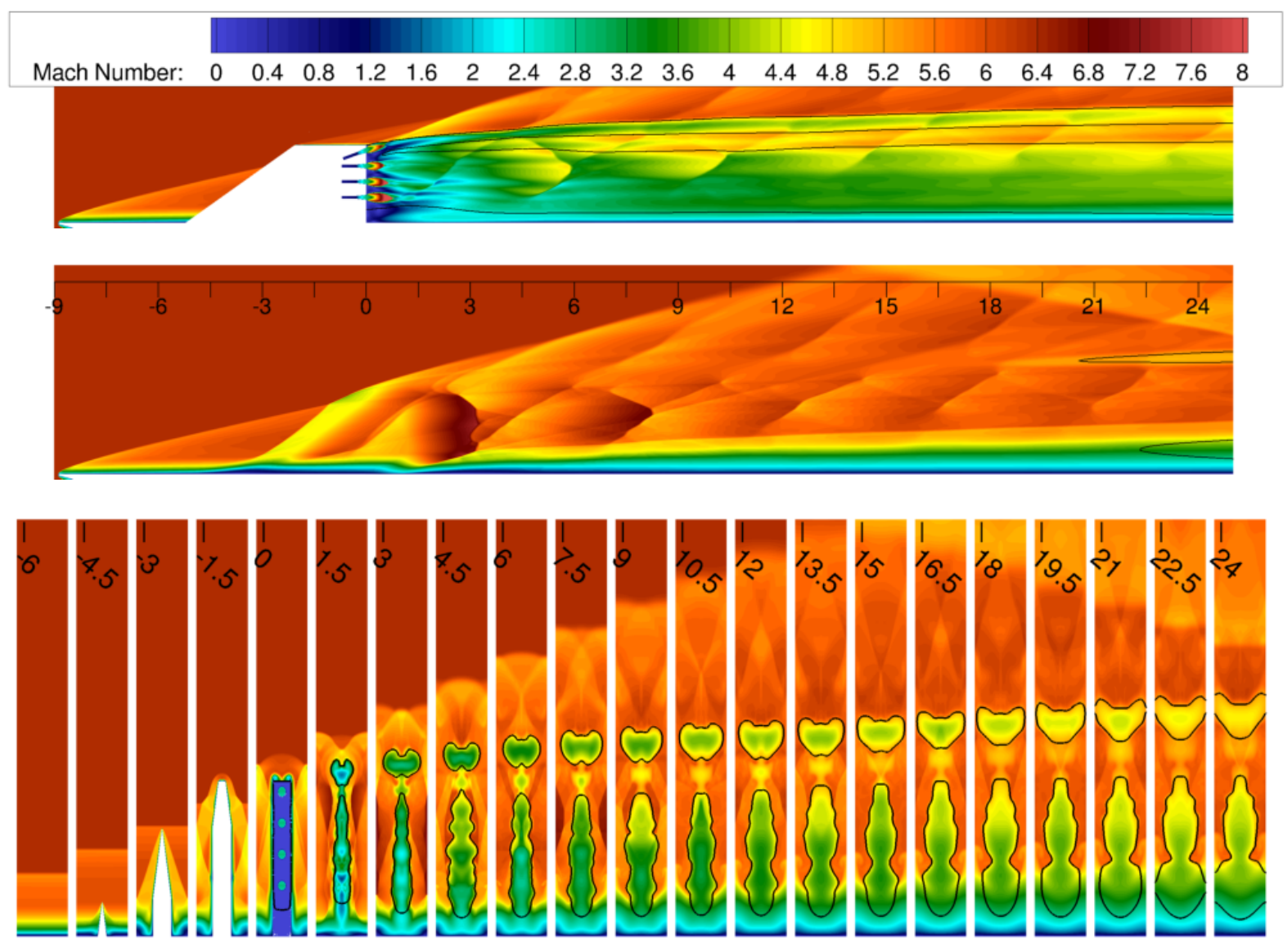

Figure 6. Contours of the Mach number on the z-planes obtained at the centerline, and half-way between the injectors, and $x$-planes at various downstream locations for the strut injector simulations. Downstream distance is in inches. Black lines denote the stoichiometric value of the fuel mass fraction.

were typically performed using 480 compute cores with most cases converging in 5 days. The total computational cost of the study was about 1 million CPU hours, which included medium and coarse mesh simulations (about $100 \mathrm{k}$ CPU hours), and pre- and post-processing of the fine mesh simulation results (about $10 \mathrm{k}$ CPU hours).

\section{Results and Discussion}

Contour plots of the Mach number in the z-planes obtained through the center of the injector ports and midway between the injectors, and $\mathrm{x}$-planes at various locations for the strut and ramp injectors for all of the cases are shown in Figs. 6-8. The flow is left-to-right. For the x-planes, the aspect ratio is not one because these planes are viewed looking aft-to-fore from an angle of about 30 degrees to the $\mathrm{x}$-axis in the xz-plane. The streamwise distance on these figures is in inches. The black isocontour line denotes a helium mass fraction equal to the stoichiometric value for hydrogen (0.0285), which closely approximates the location of the peak heat release in reacting flows and further delineates a boundary of our mixing metric of interest (Eq. (5)). The extent of mixing may be qualitatively observed by examining the extent of the area enclosed by this isocontour line. However, some care should be exercised when interpreting this area because the fuel will diffuse to its stoichiometric isocontour value only in the global sense (and after long-time mixing or in the far-field). As the fuel and air mix in the near-field of the injector, there could exist local mixing regions where the equivalence ratio is rich or lean. In the former, the area enclosed by the stoichiometric value will simply grow more slowly, but in the latter case this area will first grow and then shrink and collapse as the fuel mixes with air to locally sub-stoichiometric proportions.

Qualitatively, the flow features for all of the injectors are very similar. Upstream, the leading edge of the flat plate 

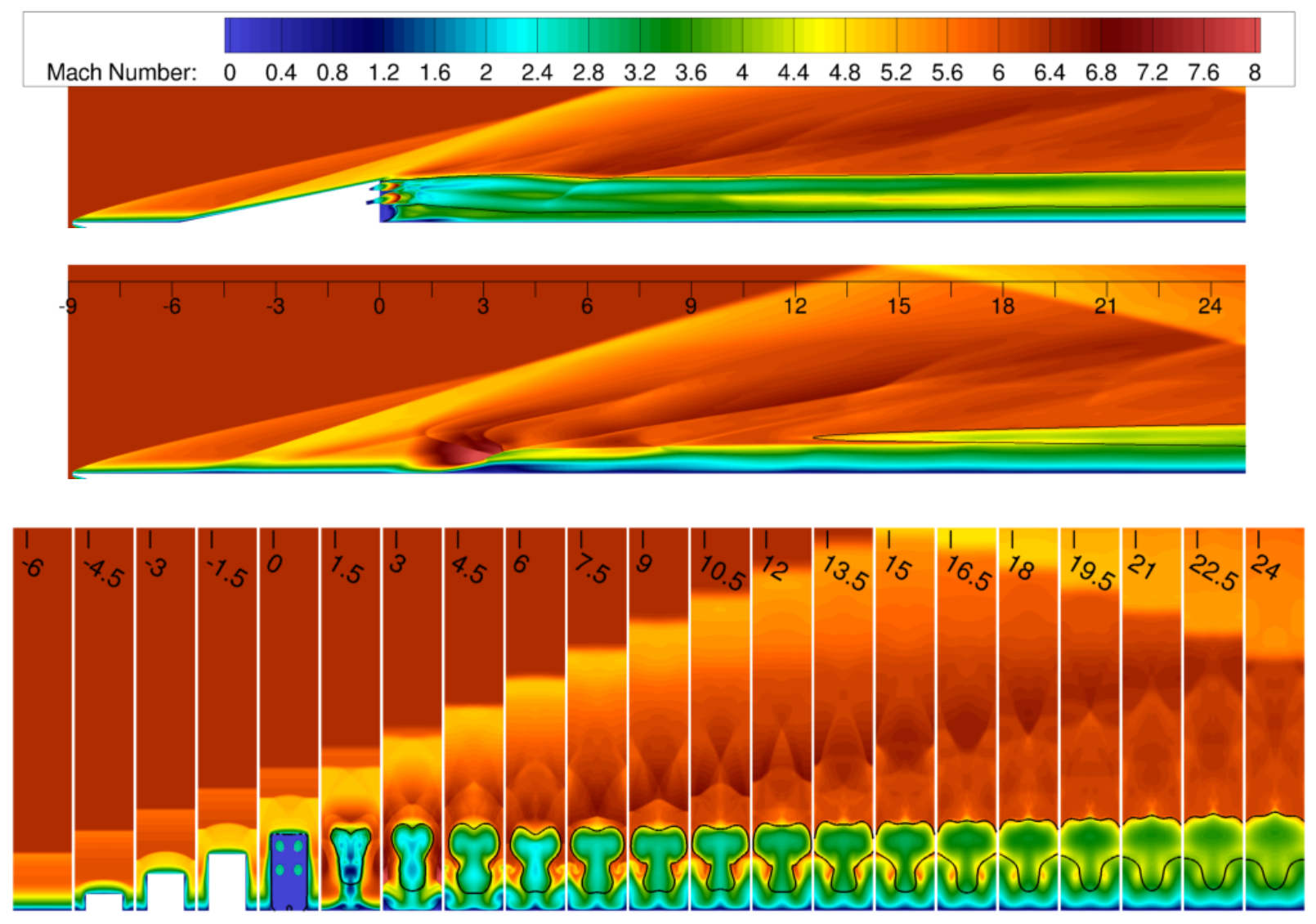

Figure 7. Contours of the Mach number on the z-planes obtained through the center of the injector ports, and half-way between the injectors, and $x$-planes at various downstream locations for the ramp injector simulations. Downstream distance is in inches. Black lines denote the stoichiometric value of the fuel mass fraction.

causes a shallow bow shock at about 12.5 degrees to the flat plate, which is slightly larger than the Mach wave angle of about 9 degrees for this Mach number. The approach boundary layer thickness is a fraction of the height of both the strut and the ramp injector bodies such that the side walls of both injectors are exposed to the free stream air flow. As a consequence, the boundary layers that develop on the sides of the injector bodies, just as the boundary layer near the leading edge of the flat plate, are thin and transitional, which makes them more susceptible to shock-induced separation. For the current turbulence model, the transition is expected to occur at a streamwise Reynolds number that is about an order of magnitude lower than that found in most turbulent flows of engineering interest. ${ }^{32}$ Therefore, the simulated flows are expected to become fully turbulent sooner than their realistic counterparts and will, therefore, be more resilient to shock-induced separation.

For the strut injector, shown in Fig. 6, a cross-stream shock wave is generated by the sharp leading edge of the strut injector body (e.g., at -3 inches). The turning half-angle of the strut injector body leading edge is 6.25 degrees (see Fig. 1), resulting in the leading edge shock wave angle of about 17 degrees as measured from the CFD. Because the leading edge of the strut body is swept at 35.4 degrees, the resulting oblique shock wave is reflected at somewhat larger angle than that expected for the turning angle of 6.25 degrees. This shock wave propagates in the cross-stream direction and impacts the body of the adjacent injector. This propagation is evident by the change in the value of the Mach number at downstream locations between -3 and 0 inches on the middle plots of each figure. After the reflection from the adjacent injector, the oblique shock waves continue to pass through one another and interact, leading to the complex downstream pattern seen in the figures. As these shock waves pass through the variable density fuel-air interface, vorticity is produced locally due to the effect of the baroclinic torque. Although the baroclinic effects are generally small, this vorticity curves and stretches the local fuel-air interface regions, thereby increasing the fuel-air 

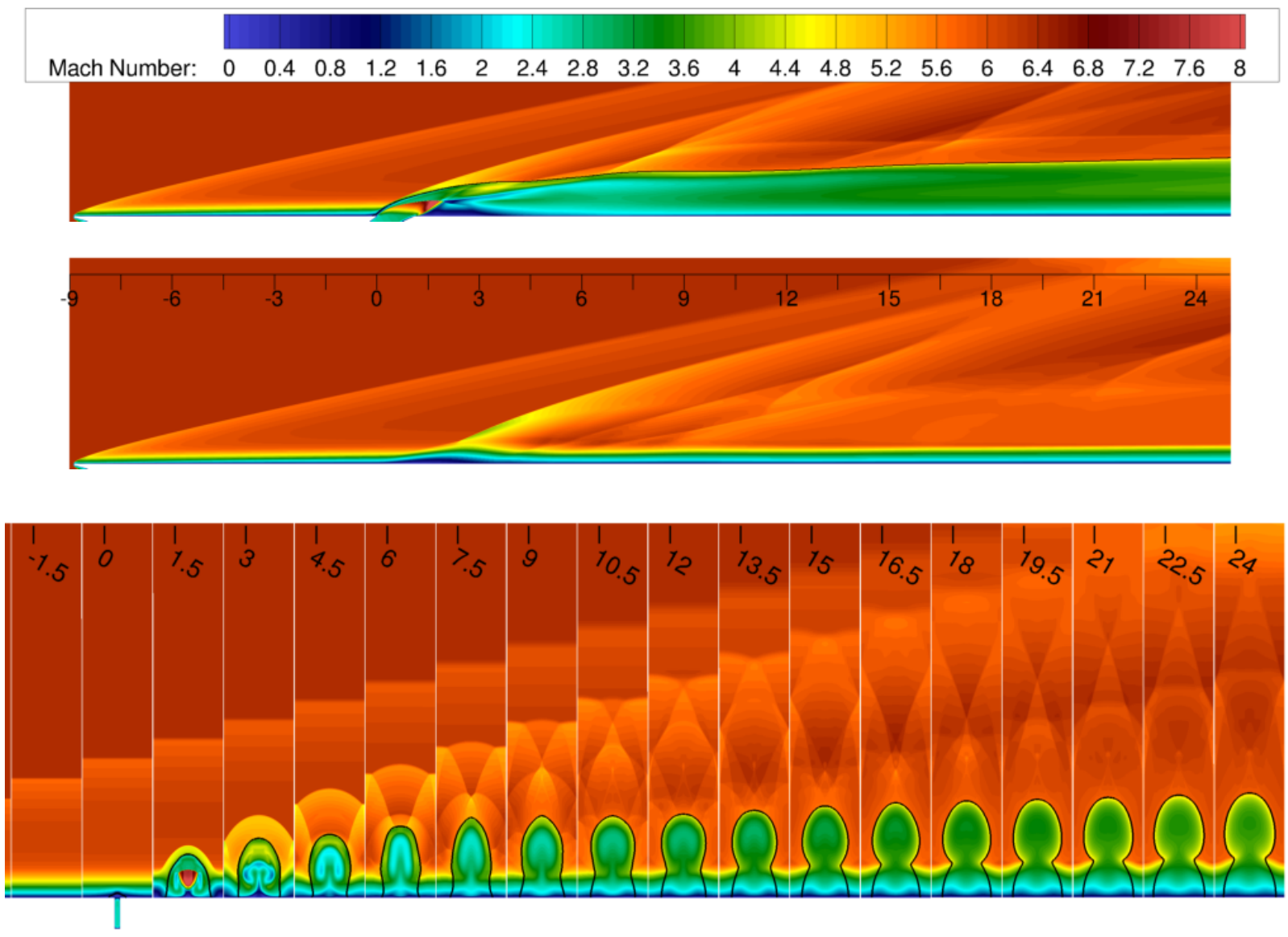

Figure 8. Contours of the Mach number on the z-planes obtained through the center of the injector port, and half-way between the injectors, and $x$-planes at various downstream locations for the flushwall injector simulations. Downstream distance is in inches. Black lines denote the stoichiometric value of the fuel mass fraction.

mixing rate, albeit at the expense of total pressure losses. As the flow continues past the strut injector body, a counter rotating vortex pair (CVP) forms near the tip of the injector body. The top-most fuel port injects the fuel stream directly at the space between the CVP. The combined effect of the angled injection and the CVP distorts and bifurcates the top-most fuel stream, and separates it from those of the three lower parallel fuel ports. This effect can be seen by observing the stoichiometric value of the mass fraction as it evolves in the x-planes in Fig. 6.

For the ramp injector, shown in Fig. 7, an oblique shock wave is generated by the inclined ramp surface of the ramp injector body. The turning angle of the ramp surface is 11.8 degrees (see Fig. 2), resulting in the oblique shock wave angle of about 17 degrees at the injector centerline. The value of this angle is slightly reduced from the expected value (of about 19 degrees) for this turning angle because of the interaction of the shock with the approach boundary layer. This oblique shock interacts and coalesces with the same shock wave produced by the adjacent injector bodies. However, unlike the cross stream shock waves that emanate from the leading edge of the strut, the ramp body oblique shock wave does not interact with the fuel-air mixing plume but instead serves primarily to introduce a pressure difference between the top of the ramp surface and the gap between the adjacent injectors. The pressure is higher on the ramp surface, which creates a driving force for the flow to spill from the ramp surface into the gap between the adjacent injectors. This spillage introduces counter rotating vortices on either side of the ramp injector body with the size proportional to the ramp height. These vortices are large compared to the size of the fuel ports. Therefore, when they begin to interact with the injected fuel streams, they stretch and push the fuel-air interface upwards away from the plate boundary layer and into the core of the flow. This process is further aided by the fact that all the ramp fuel ports are angled at 11.8 degrees up and the top set is angled at 10 degrees outwards towards the gap between the adjacent injectors. The combined effect of the angled injection and the large-scale CVP spreads the fuel through the intended 
fueling area. These effects can be seen by observing the stoichiometric value of the mass fraction as it evolves in the x-planes in Fig. 7.

For the flushwall injector, shown in Fig. 8, the flow features and dynamics are somewhat similar to that of the ramp injector. An oblique bow shock wave is generated by the fuel entering into the supersonic cross-stream. The oblique shock wave angle is about 16 degrees at the injector centerline. This bow shock interacts and joins with the same bow shock wave produced by the adjacent injector fuel plumes. Similar to the shock generated by the ramp body, the flushwall injector oblique shock wave does not significantly interact with the fuel-air mixing plume but instead serves primarily to introduce a pressure difference between the top of the fuel plume and the gap between the adjacent injectors. The pressure is higher on the top of the plume, which creates a driving force to form a CVP around the fuel plume. This CVP is too weak to significantly deform the stoichiometric isosurface, nevertheless it does provide an upward lifting force as the fuel is convected downstream. A secondary CVP also forms inside the stoichiometric isocontour line. This CVP is driven by the underexpanded fuel plume penetrating into the supersonic cross-flow and can be seen at $\mathrm{x}=3$ station on Fig. 8. Because the flushwall injector is underexpanded (see Table 1) the fuel plume spreads laterally in addition to penetrating into the cross-flow. The underexpansion process "redistributes" some of the dynamic pressure (or momentum) of the fuel jet laterally, thereby reducing the amount available for cross-flow penetration. Nevertheless, the penetration is comparable to that of the ramp injector. The primary difference between the flushwall injection and that using a strut or a ramp is that a significant amount of fuel remains in the boundary layer. The fact that the fuel contacts the wall reduces the fuel-air interface area (and mixing) as compared to a fully lifted fuel plume. Furthermore, depending on the wall temperature, the combustion could be quenched by the wall, thereby preventing the extraction of exothermic energy from the fuel. However, if combustion does occur in the boundary layer it would increase the thermal loads on the wall but at the same time provide some means of flame-holding. In addition, Chan et al. ${ }^{35}$ have reported evidence of drag reduction by near-wall burning.

Plots of the one-dimensional values of the mass-flux-weighted average Mach number, and mixing efficiency versus distance in inches are shown in Fig. 9 for the strut, ramp, and flushwall injectors. These plots help to quantify the overall behavior of the flow and the level of mixing found among all of the simulated cases. Considering the one dimensional value of the Mach number first, the results indicate that the Mach number decreases throughout the flow. The decreases are caused by the effect of the shock waves and friction losses throughout the flowpath and by the mixing losses downstream of the injection plane. The shock waves and drag losses induced by the strut and ramp injector bodies are evident by a more significant decrease in the Mach number at the injection plane, and the overall offset of values, as compared to the flushwall injector. Downstream of the injection plane the profiles of the Mach number for the strut and ramp injectors appear to follow a slightly stair-stepping pattern. This effect is caused by the shock waves crossing through, reflecting, and interacting with the mixing plume and is most pronounced in the strut injector case.

The mixing efficiency results are consistent with the observations of the Mach number contours and the corresponding areas enclosed by the stoichiometric values of the fuel species elemental mass fractions. The fuel and air mix the fastest for the strut injector, followed by the ramp, and the flushwall injectors. The profiles corresponding to the strut and ramp exhibit three distinct mixing regions. The first region, in the near-field of the injector body from 0 to about 2 inches (or about an injector body height), is characterized by a rapid rise in the mixing efficiency. The length of this region also correlates with the wake generated by the injector bodies. The fuel injected into this wake region has more time to mix with the surrounding air. This region is followed by a fairly long region where the mixing efficiency increases less rapidly and linearly with the downstream distance. The third region begins at about 22 inches and is characterized by a further decrease in the mixing rate. Unlike the strut and ramp injectors, the flushwall injector exhibits a linear increase in the mixing efficiency with the downstream distance.

Figure 10 shows the one-dimensional values of the mass-flux-weighted average axial vorticity magnitude, and the turbulence kinetic energy (TKE). The former is proportional to the circulation, which is a measure of large scale rotating motions in the cross-stream planes. The latter is an indicator of the intensity of the turbulence motions. Both quantities are indicative of the potential for the large vortical structures and the turbulence motions, respectively, to distort and wrinkle the fuel-air interface area, and enhance mixing. The strut injector body induces the largest amount of axial vorticity. This vorticity is produced near the tip of the strut, as well as, on the sides by the crossstream pressure differences induced by the complex patterns of interacting shock waves. Downstream of the strut injector body additional vorticity is produced by the expanding fuel plumes, which redistribute the initially axial momentum laterally, thereby producing additional cross-stream vorticity near the fuel-air interface. The strut injector also generates the largest amount of turbulent fluctuations, which indicates that turbulent motions are most intense for this injector. 

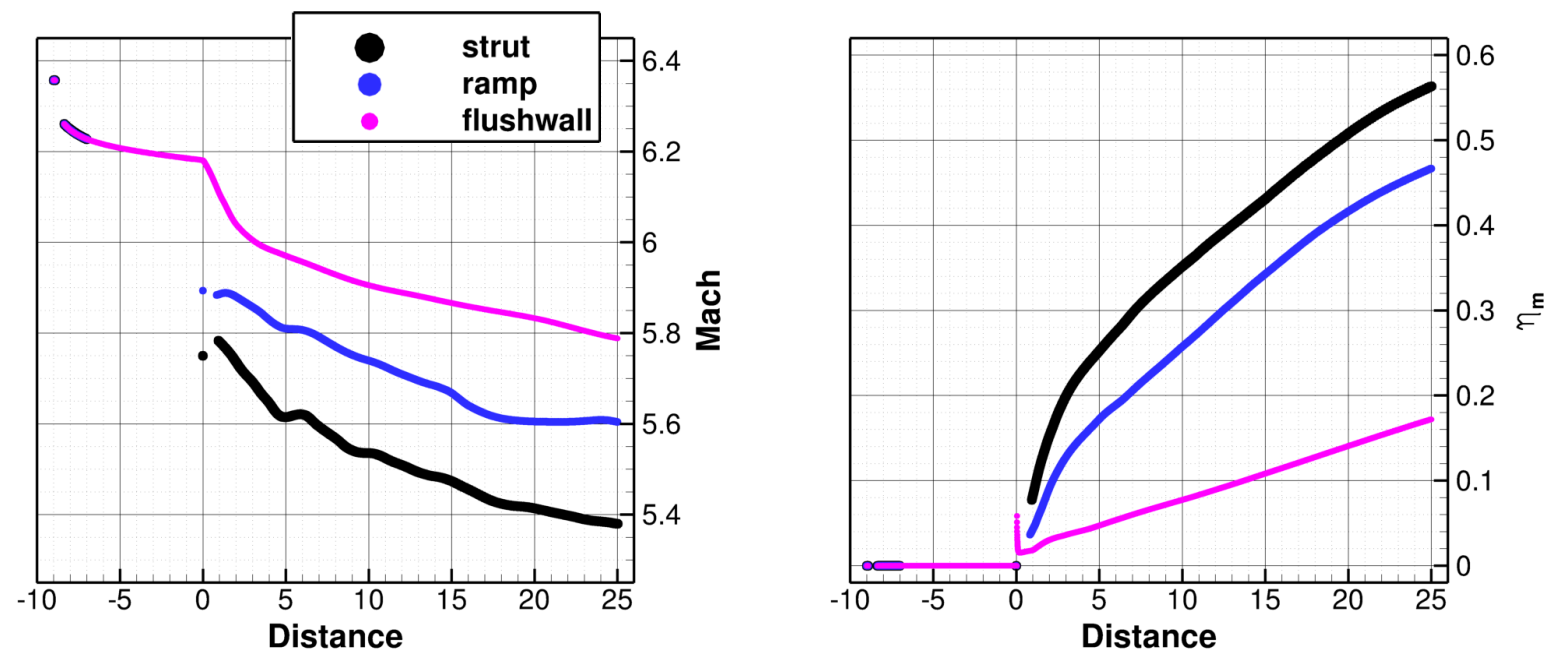

Figure 9. One-dimensional values of the Mach number, and mixing efficiencies vs. the downstream distance (in inches).
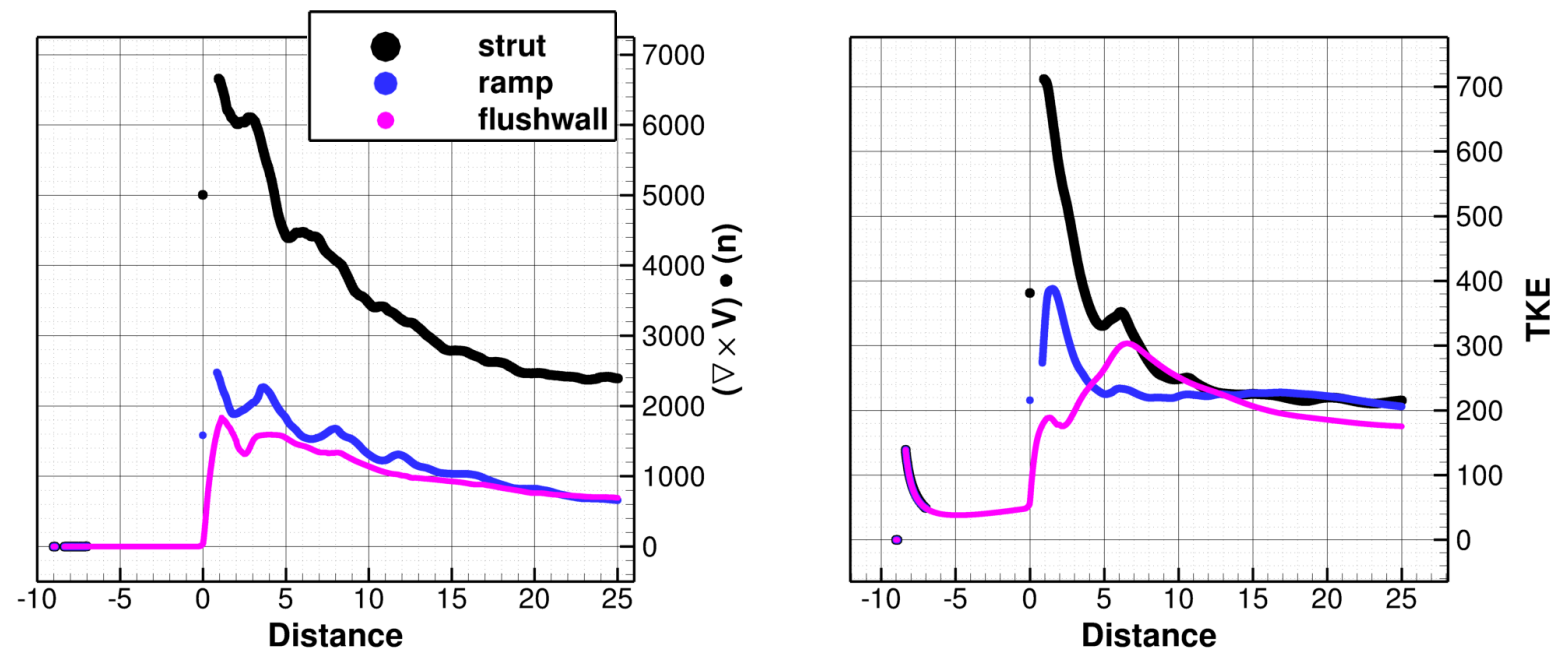

Figure 10. One-dimensional values of the axial (unit vector $n$ is oriented in the streamwise direction) vorticity magnitude (1/s), and the turbulence kinetic energy $\left(\mathrm{m}^{2} / \mathrm{s}^{2}\right)$ vs. the downstream distance (in inches).

The ramp and flushwall injectors produce comparable amounts of both axial vorticity and turbulence intensity. The axial vorticity corresponding to the ramp injector is dominated by the large scale vortex associated with the rampinduced CVP. The flushwall injector produces two relatively large CVPs, first directly behind the bow shock, and a second inside and around the fuel plume due to underexpansion (as discussed earlier). Despite a comparable amount of vortical and turbulent motions, and penetration (as see in Figs. 7, and 8), the mixing efficiency of the flushwall injector is significantly less than that for the ramp injector. This indicates that both the bluff-body flow separation and the wake produced by the ramp injector body, which increase the residence time for the fuel and air mixing, have a significant impact on the rate of mixing.

The total pressure recovery profiles are shown in Fig. 11. Because the total pressure is proportional to the entropy, 

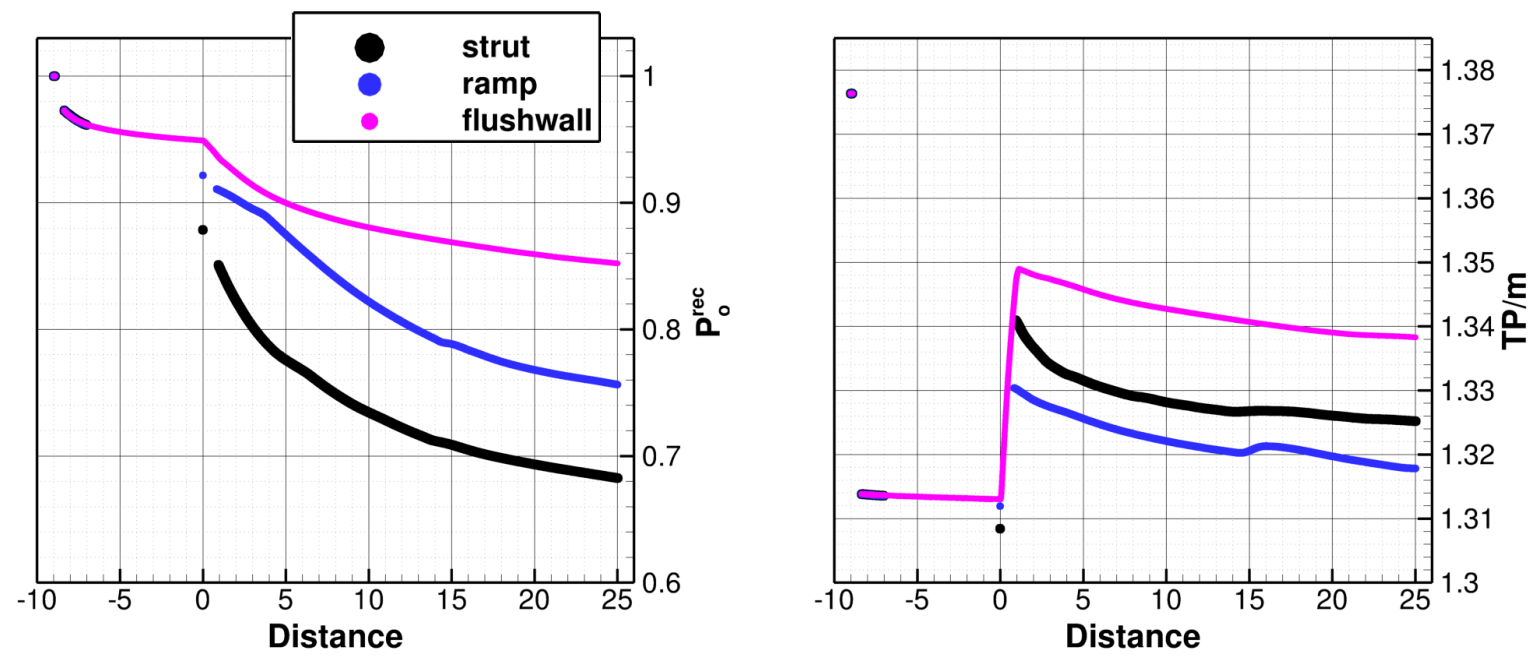

Figure 11. One-dimensional values of total pressure recovery, and the specific thrust potential $(\mathrm{kN} /(\mathrm{kg} / \mathrm{s}))$ vs. the downstream distance (in inches).

the total pressure recovery can only decrease (in adiabatic flows). The decreases are due to shock and viscous losses upstream of the injection plane and also mixing downstream. For the strut and ramp injectors, about $8 \%-16 \%$ of the total pressure losses occur upstream of the injection plane with the strut body inducing almost twice as much losses as the ramp. This is not necessarily surprising because the current strut injector exposes almost twice as much surface area to the flow as the ramp injector, thereby inducing more viscous losses. Downstream of the injection plane, the mixing losses further contribute to the decrease. In general, the losses are inversely proportional to mixing, i.e., greater mixing induces more total pressure losses. Therefore, while the flushwall injector exhibited significantly less mixing than either the strut or the ramp, it also exhibits the least amount of total pressure losses. This is a significant observation because the total pressure is proportional to the amount of momentum that can be expanded to produce thrust.

The specific thrust potential, also shown in Fig. 11, is obtained by normalizing the thrust potential by the mass flow rate of air. Unlike the total pressure recovery, the value of the thrust potential increases when heat is released into the flow by combustion. This feature makes the thrust potential a good metric of losses that are important to the engine performance, i.e., viscous and shock losses decrease the thrust potential, whereas heat release and axial fuel injection increase it. Because of these aspects of this metric, it was included even in the current non-reacting simulations. Despite the relatively small decrease of only a $2 \%-3 \%$ percentage points in the value of the total pressure recovery, the majority of the potential thrust is lost due to the shock wave induced by the leading edge of the plate. This is evident by the rapid drop in the values of the specific thrust potential just downstream from the inflow and shown in Fig. 11. Only a small fraction of the potential thrust is lost through the viscous and shock losses incurred on the injector bodies. Just downstream of the injection plane, the thrust potential is augmented by the fuel momentum. This increase occurs despite the fact that the mass flow rate of the fuel is a small fraction (FAR is 0.0292) of that of the incoming air. The amount of the thrust augmentation varies among the cases in proportion to the dynamic pressure ratio. Interesting to note is that, although the flushwall injector produces the least amount of mixing, at its 30 degrees incline, it produces the largest amount of potential thrust augmentation. The large augmentation is expected because the downstream component of the dynamic pressure ratio for the flushwall injector is about two and four times larger than that of the strut and ramp injectors, respectively.

Figure 12 shows a plot of total pressure recovery versus the mixing efficiency. On this plot, an ideal fuel injector would be represented by a nearly horizontal line with a slightly negative slope accounting for only the minimum required fuel-to-air mixing losses. For realistic injectors, viscous and shock losses further decrease the value of this slope. For the current cases, the amount of total pressure loss per unit mixing (i.e., the slope of the curve) is about the same for the strut and ramp injectors, indicating that both devices are equally "efficient" at mixing the fuel and air 


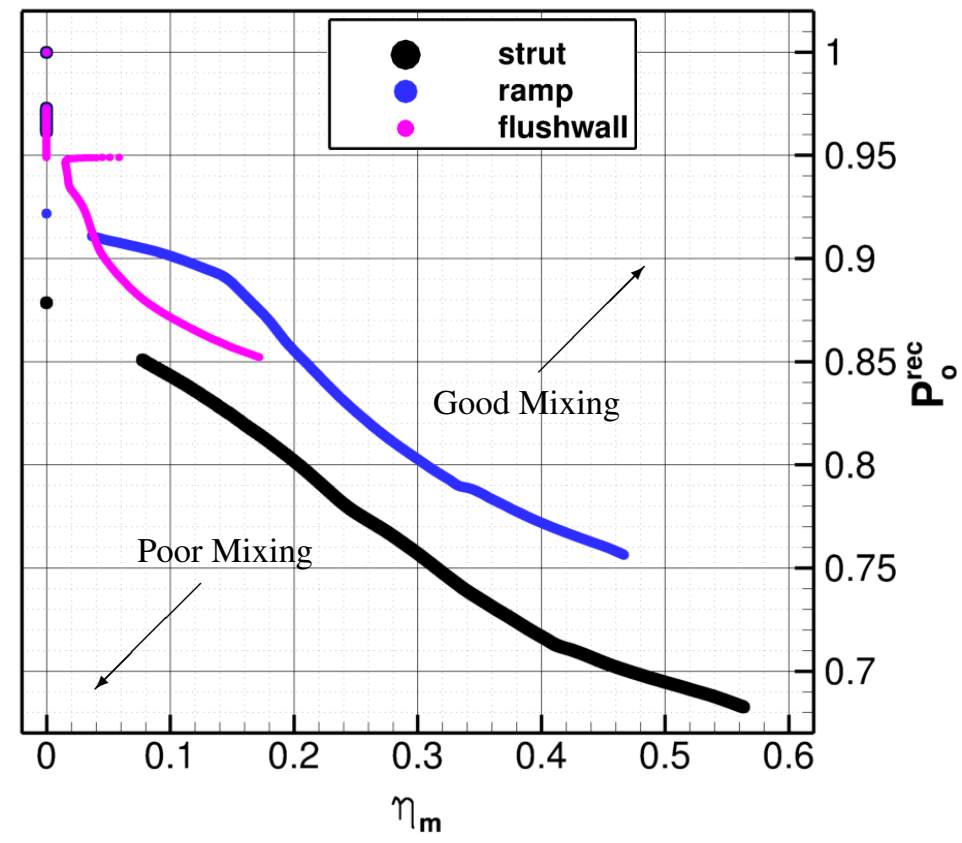

Figure 12. One-dimensional values of the total pressure recovery vs. mixing efficiency.

under the current conditions. The downward offset of strut profile is a result of the greater total pressure losses incurred on the strut injector body. Nevertheless, these larger losses are indeed converted into greater mixing, as indicated by a larger final value of the mixing efficiency for the strut as compared to both the ramp and a flushwall injectors.

The flushwall injector is less efficient at near-field mixing. The rapid decrease in the total pressure recovery for the flushwall injector includes the bow shock losses, which for the strut and ramp injectors occurred upstream of the injection plane at the leading edges of their respective injector bodies. For these injectors, the upstream losses are noted on the plot at the mixing efficiency of zero. Nevertheless, unlike the strut injector, the significant, early total pressure losses for the flushwall injector do not result in a corresponding increase in the value of the mixing efficiency. However, the final slope of the profile does approach that for the ramp and the strut, indicating a comparable rate of total pressure loss vs. mixing in the far-field.

Overall, the flushwall injector and its downstream mixing field induce less total pressure loss than those for the strut and ramp. However, the amount of mixing induced at a given downstream distance is also significantly lower, but the thrust potential is the largest. Such trade-offs, when considered together with the other factors such as combustion, which increase the thrust potential in proportion to the mixing (and combustion) efficiency, make comparisons between the different injectors quite complicated, and ultimately may prove to require considering a ducted flowpath, that includes space-filling injector arrangements, together with the knowledge of the specific flight trajectory.

\section{Conclusions}

The CFD analysis based on Reynolds-Averaged Simulations (RAS) is presented of the mixing characteristics, performance, and trade-offs of three types of fuel injectors at hypervelocity flow conditions. The injectors consist of a fuel placement device, a strut; a fluidic vortical mixer, a ramp; and a geometrically-optimized ${ }^{21}$ flushwall injector. The strut injector has a slender, swept, body protruding into the core of the flowpath, the ramp injector produces a large vortex using an unswept ramp, and the flushwall injector is a downstream-oriented high-aspect-ratio rectangle inclined to the wall to augment the thrust. These choices represent three main categories of injectors typically considered in the propulsive devices used for high-speed flight. The contours of the Mach number obtained from the RAS revealed the qualitative mixing characteristics for the three injectors. It was found that: the strut injector induced the most rapid 
mixing; the ramp-induced counter-rotating vortex pair was not sufficient to enhance upward fuel penetration, but the fuel was injected and remained above the boundary layer; and even with penetration comparable to that of the ramp, the flushwall injector introduced a significant amount of fuel into the boundary-layer. The one-dimensional values of the mass-flux-weighted average Mach number, mixing efficiencies, total pressure recovery, and specific thrust potential, as well as, mass-flux-weighted average axial vorticity, and turbulence kinetic energy, were used to quantify the differences among the injectors. These quantities showed that although the strut injector produced the greatest amount of fuelto-air mixing, it did so at the expense of significant total pressure losses. On the other hand, the flushwall injector, which produced the least amount of mixing, exhibited the greatest value of the specific thrust potential augmentation because of its large dynamic pressure. The one-dimensional plots of the total pressure recovery vs. mixing efficiency showed that the mixing "efficiency" rates (i.e., the slope of these curves) for the mixing fields corresponding to the strut and ramp injectors are comparable, and that the flushwall injector approaches this value in the far-field. The primary goal of the current work is not to pinpoint the best fuel injector for hypervelocity flow applications but rather to discuss physical considerations, and illustrate and highlight the many competing factors related to fuel injection that may impact the eventual injector performance at any flow conditions. The current analysis highlights the challenges associated with selecting the "best" injector in the presence of competing factors, i.e., maximizing mixing efficiency, total pressure recovery, and thrust potential. Future work will utilize the current injectors in ducted configurations with hydrogen fuel and chemical reactions.

\section{Acknowledgments}

Many stimulating discussions with Dr. Richard L. Gaffney Jr. on the subject of high-speed injection and mixing are gratefully acknowledged. This work is supported by the Hypersonic Airbreathing Propulsion sub-project of the Aeronautics Evaluation and Test Capabilities Project of the Advanced Air Vehicles Project in the NASA Aeronautics Research Mission Directorate (ARMD). Computational resources are provided by the NASA Langley Research Center and the NASA Advanced Supercomputing (NAS) Division.

\section{References}

${ }^{1}$ Peebles, C., Road to Mach 10: Lessons Learned From the X-43A Flight Research Program, Library of Flight Series, AIAA, Washington, D.C., 2008.

${ }^{2}$ Hank, J.M., Murphy, J.S., and Mutzman, R.C., "The X-51A Scramjet Engine Flight Demonstration Program,” in 15th AIAA International Space Planes and Hypersonic Systems and Technologies Conference, AIAA, Dayton, OH, 2008. 453-477.

${ }^{3}$ Papamoschou, D. and Roshko, A., "The Compressible Turbulent Shear Layer: An Experimental Study,” J. Fluid Mech., Vol. 197, 1988, pp.

${ }^{4}$ Givi, P., Madnia, C.K., Steinberger, C.J., Carpenter, M.H., and Drummond, J.P., "Effects of Compressibility and Heat Release in a High Speed Reacting Mixing Layer,” Combust. Sci. Technol., Vol. 78, 1991, pp. 33-68.

${ }_{5}^{5}$ Drummond, J.P., Carpenter, M.H., and Riggins, D.W., "Mixing and Mixing Enhancement in Supersonic Reacting Flow Fields," in S.N.B. Murthy and E.T. Curran, eds., High Speed Propulsion Systems, Vol. 137 of AIAA Progress Series, chap. 7, American Institute of Aeronautics and Astronautics, 1991, pp. 383-455.

${ }^{6}$ Drummond, J.P. and Givi, P., "Suppression and Enhancement of Mixing in High-Speed Reacting Flow Fields," in J.D. Buckmaster, T.L. Jackson, and A. Kumar, eds., Combustion in High-Speed Flows, Kluwer Academic Publishers, Netherlands, 1994, pp. 191-229.

${ }^{7}$ Vreman, A.W., Sandham, N.D., and Luo, K.H., “Compressible Mixing Layer Growth Rate and Turbulence Characteristics," J. Fluid Mech., Vol. 320, 1996, pp. 235-258.

${ }^{8}$ Foysi, H. and Sarkar, S., “The Compressible Mixing Layer: An LES Study,” Theor. Comp. Fluid. Dyn., Vol. 24, 2010, pp. 565-588.

${ }^{9}$ Lee, J., Lin, K.C., and Eklund, D., "Challenges in Fuel Injection for High-Speed Propulsion Systems," AIAA J., Vol. 53, No. 6, 2015, pp. 1405-1423.

${ }^{10}$ McClinton, C.R., "Evaluation of Scramjet Combustor Performance Using Cold Nonreactive Mixing Tests," in 14th AIAA Aerospace Sciences Meeting, Washington, DC, 1976.

${ }^{11}$ Drozda, T.G., Baurle, R., and Drummond, J.P., "Impact of Flight Enthalpy, Fuel Simulant, and Chemical Reactions on the Mixing Characteristics of Several Injectors at Hypervelocity Flow Conditions," in 63rd JANNAF Propulsion Meeting / 47th CS / 35th APS / 345h EPSS / 29th PSHS Joint Subcommittee Meeting, Newport News, VA, 2016.

${ }^{12}$ Baurle, R.A., Fuller, R.P., White, J.A., Chen, T.H., Gruber, M.R., and Nejad, A.S., "An Investigation of Advanced Fuel Injection Schemes for Scramjet Combustion," in 36th Aerospace Sciences Meeting and Exhibit, Reno, NV, 1998.

${ }^{13}$ Cabell, K., Drozda, T.G., Axdahl, E.L., and Danehy, P.M., "The Enhanced Injection and Mixing Project at NASA Langley," in JANNAF 46th CS / 34th APS / 34th EPSS / 28th PSHS Joint Subcommittee Meeting, Albuquerque, NM, 2014.

${ }^{14}$ Gruber, M.R., Nejad, A.S., and Dutton, J.C., "Circular and Elliptical Transverse Injection into a Supersonic Crossflow - The Role of Large-Scale Structures,” in 26th Fluid Dynamics Conference, San Diego, CA, 1995. 
${ }^{15}$ Schetz, J.A. and Billig, F.S., "Penetration of Gaseous Jets Injected into a Supersonic Stream," J Spacecraft Rockets, Vol. 3, No. 11, 1966, pp. $1658-1665$.

${ }^{16}$ Mao, M., Riggins, D.W., and McClinton, C.R., "Numerical Simulation of Transverse Fuel Injection,” in Computational Fluid Dynamics Symposium on Aeropropulsion, NASA-CP-3078, NASA, Cleveland, OH, 1990, pp. 635-667.

${ }^{17}$ Portz, R. and Segal, C., "Penetration of Gaseous Jets in Supersonic Flows," AIAA J, Vol. 44, No. 10, 2006, pp. 2426-2429.

${ }^{18}$ McClinton, C.R., "Effect of Ratio of Wall Boundary-Layer Thickness to Jet Diameter on Mixing of a Normal Hydrogen Jet in Supersonic Stream,” NASA Technical Memorandum NASA TM X-3030, NASA, Hampton, VA, 1974.

${ }^{19}$ Maddalena, L., Campioli, T.L., and Schetz, J.A., "Experimental and Computational Investigation of an Aeroramp Injector in a Mach Four Cross Flow," in AIAA 13th International Space Planes and Hypersonic Systems and Technologies Conference, AIAA 2005-3235, 2005.

${ }^{20}$ Cox-Stouffer, S., Gruber, A., and Bulman, M.J., "A Strealined, Pressure-Matched Fuel Injector for Scramjet Applications," in 36th AIAA/ASME/SAE/ASEE Joint Propulsion Conference and Exhibit, AIAA 2000-3707, AIAA, Huntsville, AL, 2000.

${ }^{21}$ Ogawa, H., "Physical Insight into Fuel-Air Mixing for Upstream-Fuel-Injected Scramjets via Multi-Objective Design Optimization," $J$ Propul Power, Vol. 31, No. 6, 2015, pp. 1505-1523.

${ }^{22}$ Cabell, K.F. and Rock, K.E., "A Finite Rate Chemical Analysis of Nitric Oxide Flow Contamination Effects on Scramjet Performance," Tech. Rep. TP-212159, NASA, 2003.

${ }^{23}$ Brown, G.L. and Roshko, A., "On Density Effects and Large Structure in Turbulent Mixing Layers," J. Fluid Mech., Vol. 64, 1974, pp. $775-816$.

${ }^{24}$ Bogdanoff, D.W., "Compressibility Effects in Turbulent Shear Layers," AIAA J., Vol. 21, No. 6, 1983, pp. 926-927.

${ }^{25}$ Riggins, D.W., McClinton, C.R., and Vitt, P.H., "Thrust Losses in Hypersonic Engines Part 1: Methodology," J. Propul. Power, Vol. 13, No. 2, 1997, pp. 281-287.

${ }^{26}$ VULCAN-CFD, “http://vulcan-cfd.larc.nasa.gov/," 2016.

${ }^{27}$ van Leer, B., "Towards the Ultimate Conservative Difference Scheme. V: A Second-Order Sequel to Godunov's Method," J. Comput. Phys., Vol. 32, No. 1, 1979, pp. 101-136.

${ }^{28}$ Edwards, J.R., “A Low-Diffusion Flux-Splitting Scheme for Navier-Stokes Calculations,” Comput. Fluids., Vol. 26, No. 6, 1997, pp. 635659.

${ }^{29}$ McBride, B.J., Gordon, S., and Reno, M.A., "Thermodynamic Data for Fifty Reference Elements," NASA Technical Paper 3287/REV1, NASA, Cleveland, OH, 2001.

${ }^{30}$ Pulliam, T.H. and Chaussee, D.S., "A Diagonal Form of an Implicit Approximate-Factorization Algorithm,” J. Comput. Phys., Vol. 39, No. 2, 1981, pp. 347-363.

${ }^{31}$ Menter, F.R., "Two-Equation Eddy-Viscosity Turbulence Models for Engineering Applications,” AIAA J., Vol. 32, No. 8, 1994, pp. 15981605.

${ }^{32}$ Wilcox, D.C., Turbulence Modeling for CFD, DCW Industries, Inc., La Cañada, CA, 2000.

${ }^{33}$ Drozda, T.G., Axdahl, E.L., and Cabell, K.F., "Pre-Test CFD for the Design and Execution of the Enhanced Injection and Mixing Project at NASA Langley Research Center," in JANNAF 46th CS / 34th APS / 34th EPSS / 28th PSHS Joint Subcommittee Meeting, Albuquerque, NM, 2014.

${ }^{34}$ Roache, P.J., Verification and Validation in Computational Science and Engineering, Hermosa Publishers, 1998.

${ }^{35}$ Chan, W.Y.K., Mee, D.J., Smart, M.K., and Turner, J.C., "Drag Reduction by Boundary-Layer Combustion: Influence from Disturbances Typical of Cross-Stream Fuel-Injection," J Propul Power, Vol. 31, 2015, pp. 1486-1491. 